\title{
BMJ Open Efficacy of a guided internet-based intervention (iSOMA) for somatic symptoms and related distress in university students: study protocol of a randomised controlled trial
}

\author{
Severin Hennemann, ${ }^{1}$ Katja Böhme, ${ }^{1}$ Harald Baumeister, ${ }^{2}$ Eileen Bendig, ${ }^{2}$ \\ Maria Kleinstäuber, ${ }^{3}$ David Daniel Ebert, ${ }^{4}$ Michael Witthöft ${ }^{1}$
}

To cite: Hennemann S, Böhme K, Baumeister $\mathrm{H}$, et al. Efficacy of a guided internetbased intervention (ISOMA) for somatic symptoms and related distress in university students: study protocol of a randomised controlled trial. BMJ Open 2018;8:e024929. doi:10.1136/ bmjopen-2018-024929

\section{- Prepublication history for} this paper is available online. To view these files, please visit the journal online (http://dx.doi. org/10.1136/bmjopen-2018024929).

Received 27 June 2018 Revised 18 0ctober 2018 Accepted 20 November 2018

Check for updates

(c) Author(s) (or their employer(s)) 2018. Re-use permitted under CC BY-NC. No commercial re-use. See rights and permissions. Published by BMJ.

For numbered affiliations see end of article.

Correspondence to

Severin Hennemann;

s.hennemann@uni-mainz.de

\section{ABSTRACT}

Introduction Persistent and distressing somatic symptoms are common in younger age cohorts such as university students. However, the majority does not receive adequate psychosocial care. Internet-based and mobile-based interventions may represent low threshold and effective extensions to reduce somatic and associated mental symptom severity. The planned study aims to investigate the feasibility and efficacy of an internetbased intervention in reducing somatic and psychological symptoms in an international population of university students with somatic symptom burden.

Methods and analysis This parallel two-armed randomised controlled trial evaluates an 8-week guided intervention, including web-based consecutive modules based on cognitive behavioural therapy (CBT) principles against a waitlist control group. Guidance will be provided by trained psychologists with weekly written supportive feedback. As part of the 'Studicare' project, the present study aims to recruit $n=154$ university students indicating somatic symptom burden at baseline in German-speaking universities. Self-report assessments will take place at baseline and after intervention completion $(8,16$ weeks after randomisation). The primary outcome will be the severity of somatic symptoms and associated mental distress. Secondary outcomes include depression, (health) anxiety, disability, intervention satisfaction and adherence. Ethics and dissemination Ethics approval has been granted. Results from this study will be published in peer-reviewed journals and presented at international conferences.

Trial registration number DRKS00014375; Pre-results.

\section{INTRODUCTION}

The experience of somatic symptom distress represents the hallmark of a number of clinical conditions such as somatoform disorders (Diagnostic and Statistical Manual of Mental Disorders (DSM)-IV, 10th revision of the International Statistical Classification of Diseases and Related Health Problems, ICD-10), the recently adapted diagnoses under the term
Strengths and limitations of this study

- First internet-based guided cognitive behavioural therapy (CBT) for young adults with somatic symptom distress.

- International sample of German-speaking university students will be recruited.

- Challenges of this trial include the diverse target group and somatic conditions.

- Strong theoretical and clinical foundation through established CBT manual.

- Therapeutic alliance and side effects will be monitored.

somatic symptom disorders (DSM-5) as well as functional somatic syndromes (eg, fibromyalgia, irritable bowel syndrome). In all these conditions, the experience of somatic symptoms is commonly associated with high levels of impairment in quality of life, psychological distress or increased healthcare utilisation. ${ }^{12}$ Somatic symptom disorders (SSDs) are among the most prevalent disorders in primary care patients. ${ }^{3}$ Eventually, this condition has been associated with an elevated risk for chronic manifestation and comorbid mental disorders, ${ }^{4}$ work $\operatorname{loss}{ }^{5}$ or early retirement. ${ }^{6}$

Regarding the development of chronic somatic symptoms, recent integrative models highlight the role of cognitive processes beyond somatic pathologies. According to Brown, ${ }^{7}$ two attention systems regulate the experience and consequences of physical complaints: first, afferent somatic sensations are selected automatically based on heuristics from previous experiences (eg, illness, emotional distress). Second, intentional attentional processes lead to a chronic focus on these sensations, which accelerates somatic symptom perception, increases arousal and 
thus modulates the sensitivity of the primary attention system. Similarly, van den Bergh $e t a l^{8}$ highlight the probabilistic and inferential nature of information processing of somatic sensations. With regard to learning mechanisms, a series of experiments could furthermore demonstrate that somatic symptoms can be conditioned without presence of any somatic pathology, in which negative affect plays a central role. ${ }^{9}$ This interaction of physiological and psychological processes has important implications for the treatment of chronic somatic symptoms. Thus, treatment guidelines recommend a biopsychosocial rationale, combining medical care and psychosocial interventions such as psychotherapy to promote coping mechanism, functioning and quality of life. ${ }^{10}$

A growing body of evidence suggests the effectiveness of cognitive behavioural therapy (CBT) in SSDs with small-to-medium effect sizes ${ }^{11}{ }^{12}$ while the evidence for peripheral interventions (eg, medication, injections, operations) is controversial. ${ }^{13} 14$ Furthermore, self-help interventions have been shown to be effective in various somatoform disorders. ${ }^{15}$

However, Wittchen and Jacobi ${ }^{16}$ estimate that only $40 \%$ of patients with severe SSDs receive adequate psychotherapeutic care, which represents the second highest rate of undertreatment of mental disorders in Germany. Regarding the academic context, only $16.4 \%$ of university students with mental disorders in 21 countries receive adequate healthcare treatment. ${ }^{17}$ Beyond structural limitations regarding healthcare resources and treatment allocation, challenges of psychosocial care include long waiting times ${ }^{18}$ time-consuming and distressing treatment seeking, ${ }^{19}$ somatic-dominated health models, fear of stigmatisation, ${ }^{20}$ unfavourable treatment experiences and expectations in patients ${ }^{21}$ and health professionals' negative attitudes towards patients with somatoform disorders. ${ }^{22}$

In an effort to reduce the threshold to the access to healthcare instruments, internet-based and mobile-based interventions (IMIs) have been developed extensively within the last decade. Advantages of IMIs include widespread accessibility, local and temporal flexibility, cost-effectiveness ${ }^{23}$ and low threshold for psychological issues. ${ }^{24}$ In this regard, various studies show that IMIs are acceptable in university students. ${ }^{25}{ }^{26}$ IMIs may vary regarding technical implementation (eg, web-based or mobile-based), intervention strategy and localisation in the healthcare process (eg, prevention, stand-alone interventions, aftercare) or the amount of human guidance. ${ }^{27}$ Meta-analytic evidence indicates the efficacy of IMIs for various mental disorders with effect sizes ranging from standardised mean difference $(\mathrm{SMD})=0.31 \quad(\mathrm{n}=16)$ for eating disorders to $\mathrm{SMD}=1.52$ $(n=6)$ for panic disorders, with higher effect sizes for guided interventions. ${ }^{28}$ Further evidence suggests the efficacy of internet interventions in university students, with mediumto-high effect sizes for common mental disorders such as depression or anxiety ${ }^{29}$ and stress-reducing interventions. ${ }^{30}$

Beyond the academic context, evidence on the efficacy of IMIs in chronic somatic conditions shows a wide range of effects depending on the condition under study. Meta-analytic evidence by van Beugen $e t a l^{31}$ points to an overall small effect size for psychological outcomes (eg, depressive symptoms, $\mathrm{n}=15, \mathrm{SMD}=0.21,95 \% \mathrm{CI}: 0.08$ to 0.34 ) and mostly positive effects on disease-specific physical outcomes $(59 \%, \mathrm{n}=10 / 17$ studies in favour of internet-based CBT (iCBT)). Existing evidence mostly refers to functional somatic syndromes. ${ }^{32-34}$ Here, the evidence is promising for irritable bowel syndrome (IBS). For example, subgroup analyses of six web-based self-help interventions by Liegl et $a l^{35}$ yielded a large pooled effect size ( $\mathrm{SMD}=1.01 ; 95 \%$ CI: 0.78 to 1.23 ). The evidence base for the effectiveness of IMIs in fibromyalgia is less profound but indicates a positive effect of IMIs. ${ }^{36}$ Furthermore, a series of studies by Hedman et al with hypochondriac patients could demonstrate a large effect $(\mathrm{SMD}=1.62,95 \% \mathrm{CI}$ : 1.10 to 2.10$)$ of a 3-month web-based CBT compared with an attention control group. ${ }^{37}$ For chronic pain, a recent meta-analysis of 11 studies by Macea $e t a l^{38}$ found a small effect of web-based CBT (SMD=0.29, 95\% CI: 0.15 to 0.42 ). In chronic physical conditions, reviews indicate a lower efficacy of IMIs for distress or psychological outcomes than for somatic symptoms. ${ }^{32} 33$ Above that, studies investigating IMIs for SSD are scarce: Hedman $e t a l^{9}$ investigated a guided $\mathrm{iCBT}$ in a mixed sample including participants with SSD $(\mathrm{n}=114 / 86.4 \%)$ and yielded large effect on health anxiety (SMD=1.27, 95\% CI: 0.72 to 1.79) but not for depression in comparison to a waitlist control group (WLCG). Similarly, an Australian study testing a guided iCBT against a psychoeducation control group found high effects on the reduction of health anxiety in a sample including participants with SSD. ${ }^{40}$ However, these studies focused on health anxiety and as such did not assess the level of somatisation, limiting their generalisability to SSDs.

Thus, SSDs or somatoform syndromes remain an insufficiently studied research area in internet interventions, particularly in younger age cohorts, where elevated somatisation and elevated risk for the development of SSDs has been observed. ${ }^{41}{ }^{42}$ University students, in particular, show a higher risk of developing mental disorders than non-students, with increasing rates of severe mental health issues ${ }^{1743}$ and reduced uptake of medical services. ${ }^{44}$ A survey by Bailer $e t a t^{45}$ found that $9.1 \%$ of university students of a major German university fulfilled the criteria for a subclinical somatoform syndrome. Also, SSDs represent the second most prevalent mental disorders $(6.6 \%)$ in German university students according to an insurance report. ${ }^{46}$ Longitudinal data also indicate that stress factors contribute to the development and persistence of mental disorders in academia ${ }^{478}$ and that these have been associated with reduced academic functioning ${ }^{49}$ or university attrition. ${ }^{48}$ Together, these reports underline the importance of effective and accessible healthcare instruments in academia. 


\section{Objectives}

This trial thus aims to evaluate the feasibility and efficacy of a guided iCBT, based on an established short-term CBT manual, ${ }^{50}$ as a stand-alone transdiagnostic intervention for somatic symptoms and related distress (internet-based intervention for somatic symptoms (iSOMA)) in an adult population of university students. The present study is embedded in the project 'studicare' and hosted on the corresponding platform www.studicare.com. Studicare combines IMIs for various mental and behavioural issues (eg, procrastination, stress, mindfulness, social and test anxiety, physical activity, internet addiction, depression) to promote mental health in graduate students. It is expected that:

1. iSOMA is effective in reducing the severity of somatic symptoms and related distress compared with a WLCG.

2. Compared with WLCG, iSOMA is superior in reducing comorbid mental symptoms (depression, anxiety and health anxiety) and in improving the level of functioning and attitudes towards psychotherapy.

Furthermore, it will be explored, if the efficacy of iSOMA is moderated/mediated by somatosensory amplification, emotional reactivity, expectations towards the internet intervention, therapeutic alliance as well as medical and demographic characteristics. The influence of acceptability and intervention adherence on clinical outcomes will be examined. Possible adverse events and side effects will be monitored. Also, outcomes will be evaluated exploratively by comparing two levels of therapist guidance (regular feedback vs feedback on demand) as applied in the two intervention arms (figure 1) and the effect of optional short text messages.

\section{METHODS}

\section{Study design}

This project is a two-armed, randomised controlled study of paralleled design comparing the efficacy of an iCBT with a WLCG. Both conditions will have unrestricted access to treatment as usual. The trial will be reported in accordance with the Consolidated Standards of Reporting Trials guidelines for randomised controlled trials ${ }^{51}$ and recommendations for internet intervention trials. ${ }^{52}$

\section{Eligibility criteria}

In order to be eligible for the study, participants need to be (a) aged $\geq 18$ years, (b) enrolled university students with (c) internet access and (d) sufficient knowledge of the German language, who (e) score in a clinical range of somatisation, as indicated by a value of $\geq 4$ in the Patient

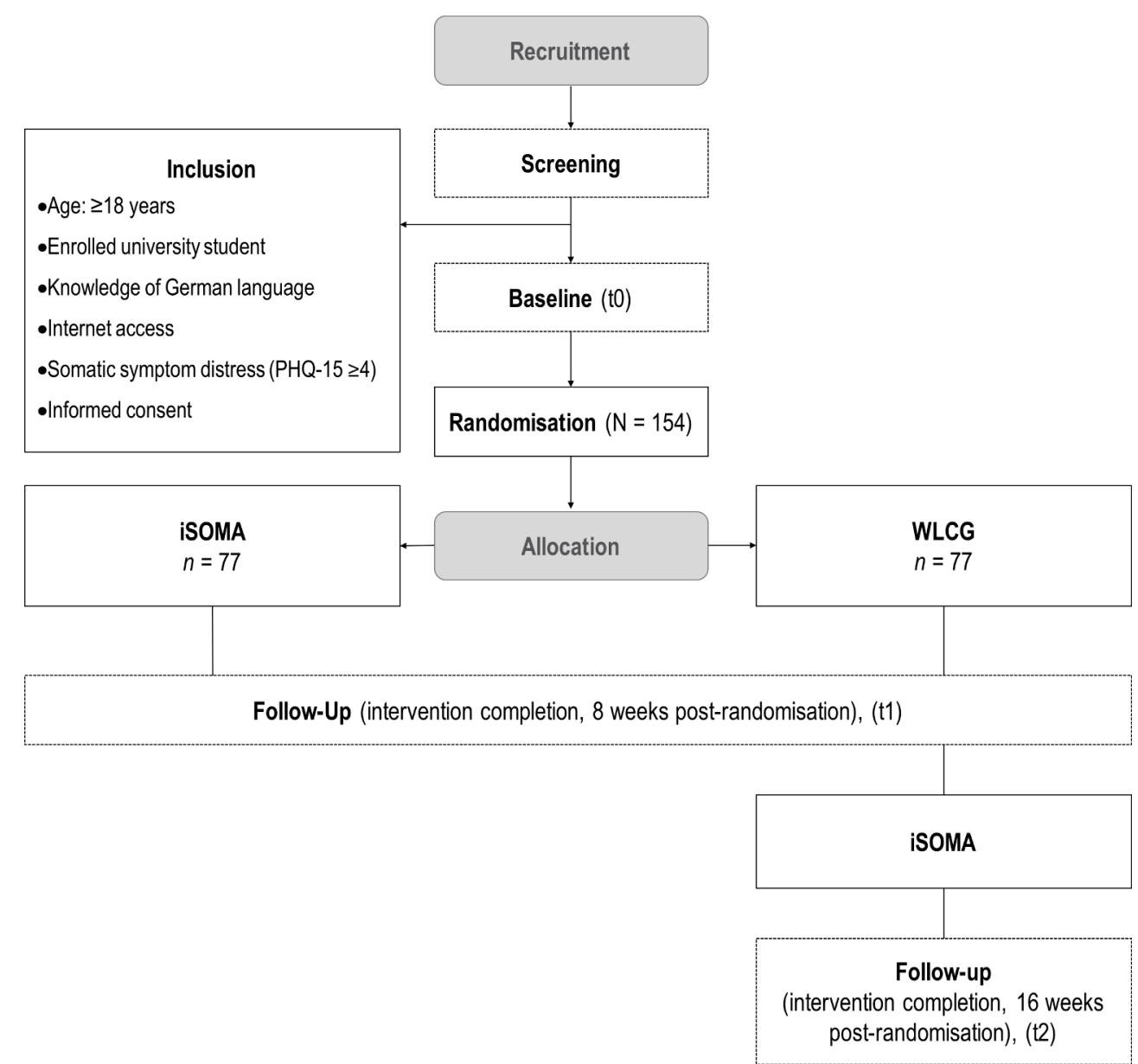

Figure 1 Flow chart of inclusion and study procedure. iSOMA, internet-based training for somatic symptoms; PHQ15, Patient Health Questionnaire; WLCG, waitlist control group. 
Health Questionnaire (PHQ-15), ${ }^{53}$ which has recently been shown to be a sensitive cut-off criterion for the detection of somatic symptom disorder. ${ }^{54}$ Furthermore, (f) participants will need to provide a signed copy of the informed consent form via email or postal.

\section{Setting/recruitment}

Recruitment has started in August 2018 and will continue until the targeted sample size has been reached. Participants from Germany, Swiss or Austrian universities are recruited consecutively through online resources (eg, studicare platform, study web portal, social networks), newspapers or university press reports and academic institutions for student health (eg, student counselling, occupational health management, college sport, etc). Recruiting measures will be applied adaptively depending on the inclusion rate. At the time of this protocol, 15 universities were already associated with studicare (see http:/ /www.studicare.com/studicare-universitaeten), sending healthcare information including IMI offers to their students on a regular basis throughout study terms. Participants can apply via email and receive screening questionnaires to assess eligibility. Eligible individuals providing their informed consent are then contacted by the study team via email including further information and a link referring to the internet intervention. Participants are informed that the intervention under study does not replace any medical diagnosis or therapy and are recommended to seek medical counselling in case of unclear health conditions.

\section{Patient and public involvement}

Patients and the public were not involved in the design of the study.

\section{Randomisation}

Eligible participants will be randomly allocated to either the internet intervention or WLCG. Randomisation and allocation will be performed by an independent person blinded to investigators and all processes within the intervention. For randomisation, an automated, web-based programme (www. sealedenvelope.com) will be used, with an allocation ratio of 1:1 and permuted block sizes $(2,4$, 6). Allocation concealment will be ensured, as the service will not release the randomisation code until the participant has been recruited into the trial, which takes place after completion of the baseline measurement. Within the course of the study, neither participants nor the e-coaches can be blinded to allocation due to the nature of the intervention. An employee outside the research team will feed data into the computer in separate datasheets so that the researchers can analyse data without having access to information about the allocation.

\section{Intervention}

Intervention condition

The intervention was adapted from an established CBT rationale for medically unexplained or somatoform symptoms ${ }^{50}$ and regarding the study design and intervention

\begin{tabular}{|c|c|c|}
\hline & Module & Content \\
\hline 1 & Goal setting & $\begin{array}{l}\text { History of symptom } \\
\text { development, goal setting }\end{array}$ \\
\hline 2 & Stress education & $\begin{array}{l}\text { Psychoeducation on stress } \\
\text { reaction, relaxation techniques }\end{array}$ \\
\hline 3 & Attention control & $\begin{array}{l}\text { Attention shift, euthymic } \\
\text { activities }\end{array}$ \\
\hline 4 & Illness attitudes & $\begin{array}{l}\text { Cognitive modification, } \\
\text { interoceptive exposure }\end{array}$ \\
\hline 5 & Illness behaviour & $\begin{array}{l}\text { Activity training, reduction of } \\
\text { avoidance or safety behaviour }\end{array}$ \\
\hline 6 & Stress management & $\begin{array}{l}\text { Transactional stress model, }{ }^{117} \\
\text { stress management techniques }\end{array}$ \\
\hline 7 & $\begin{array}{l}\text { Summary and } \\
\text { planning }\end{array}$ & $\begin{array}{l}\text { Biopsychosocial explanatory } \\
\text { model, summarising personal } \\
\text { coping strategies, relapse } \\
\text { prevention }\end{array}$ \\
\hline
\end{tabular}

structure from previous trials of the research group. ${ }^{5556}$ The intervention consists of seven, weekly modules following a short introduction. The duration of the main modules is approximately $45-60 \mathrm{~min}$. After completion, all modules are unlocked for repeated view. Each module includes psychoeducation, behavioural experiments, exercises and assignments, questions and interactive elements (eg, quizzes, conditional content) via text, audio or video. The modules start with a reflection on the assignments from the previous module, followed by an overview of the content of the present module. Table 1 summarises the topics and content of each module.

\section{Guidance}

Within 2 weekdays after module completion, participants individually receive a semi-standardised feedback from a trained psychologist (e-coach). The supportive feedback focuses on participant's entries and weekly assignments and aims to reinforce self-efficacy and adherence. ${ }^{57}$ E-coaches will send reminders to inactive participants or in case of belated module completion. Participants are able to contact the assigned e-coach any time via the internet platform and vice versa. Communication runs exclusively on the internet platform. E-coaches are clinical psychologists (undergraduate students, postgraduate clinical psychologists in psychotherapy training), who are trained beforehand and supervised regularly by experienced, licensed psychotherapists (SH, MW).

\section{Text messages}

The web-based content will be extended by optional, motivating text messages (SMS-coach). This includes regularly sent automatic text messages, which are tailored to the intervention content. Previous trials have shown the augmenting effect of text messages on efficacy and adherence. ${ }^{58}$ The messages include reminders to homework 
Table 2 Outcome criteria, measurement instrument, assessment points

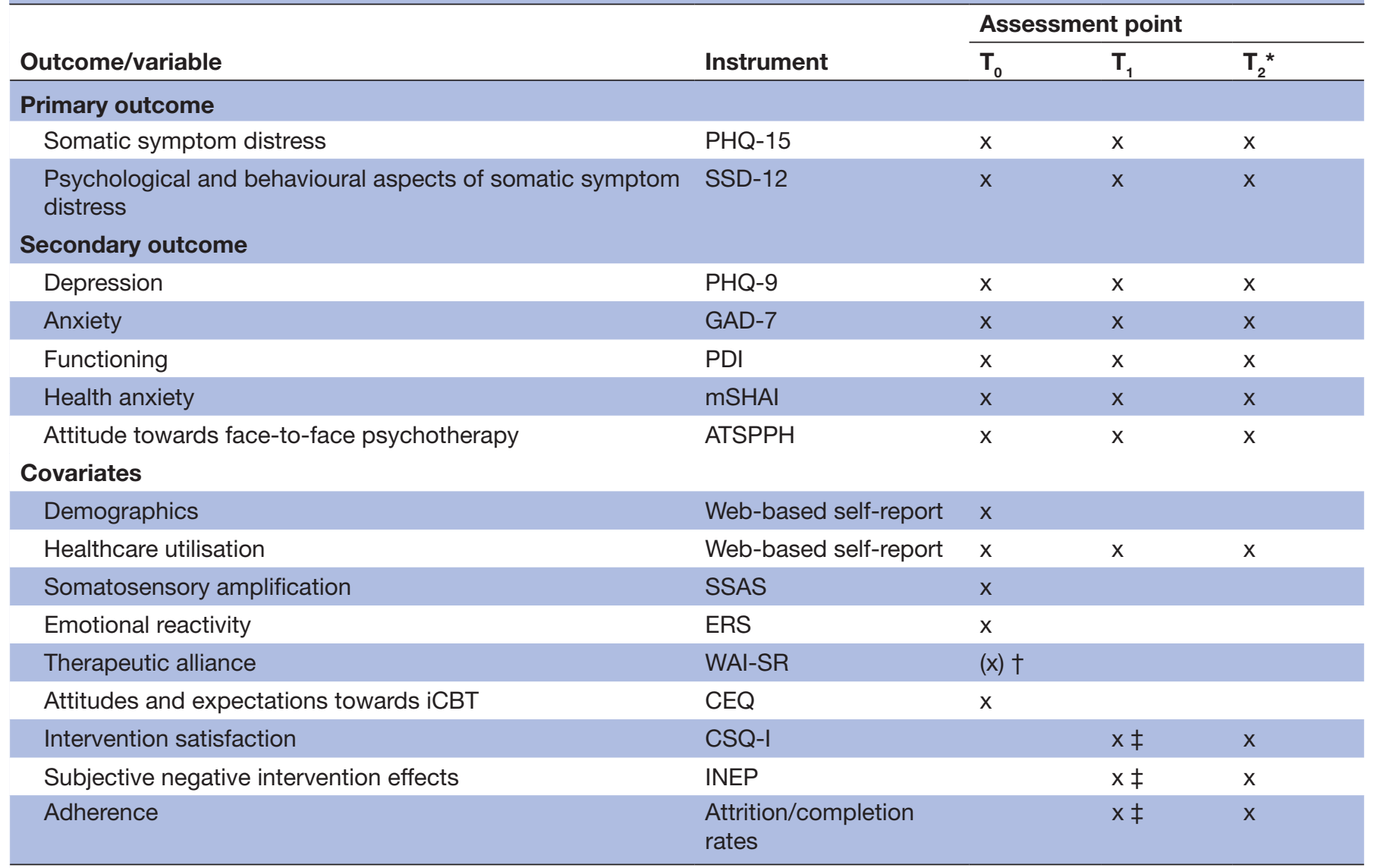

${ }^{*}$ Only for control group.

†3 weeks after intervention start.

$\ddagger$ Only for intervention group.

$T_{0}$, baseline; $T_{1}, 8$ weeks postrandomisation; $T_{2}, 16$ weeks postrandomisation; ATSPPH, Attitudes Towards Seeking Professional Psychological Help; GAD-7, Generalised Anxiety Disorder Questionnaire; INEP, Side Effects of Psychotherapy Inventory; mSHAl, modified short version of the Health Anxiety Inventory; PERS, Perth Emotion Reactivity Scale; PDI, Pain Disability Index; PHQ, Patient Health Questionnaire; SSAS, Somatosensory Amplification Scale; SSD-12, somatic symptom disorder-B Criteria Scale; WAI-SR, Working Alliance Inventory-short revised; CEQ, Credibility/Expectancy Questionnaire; CSQ-I, Client Satisfaction Questionnaire for internet-based interventions.

or intervention use, tiny-tasks or reinforcement of motivation to exercise.

\section{Control condition}

Participants in the WLCG have unrestricted access to usual treatment options. Eight weeks postrandomisation, the WLCG will receive iSOMA with written e-coach contact on demand through the intervention platform, as proven to be effective in previous trials. ${ }^{6061}$

\section{Assessments and outcomes}

Assessments will be conducted at baseline $\left(\mathrm{T}_{0}\right)$ after completion of the intervention follow-up ( 8 weeks, $\mathrm{T}_{1}$ ), respectively at 16 weeks follow-up $\left(T_{2}\right)$ for the WLCG. A list of outcomes and measurement instruments is presented in table 2. Eligibility criteria, outcomes, potential effect-modifying variables and demographics will be assessed via self-report with validated questionnaires (approximately 20-30 min per assessment). In case of non-compliance, up to three reminders will be sent via email within the week after invitation for the assessment, followed by contact via phone, after which participants will be characterised as non-completers. The instruments will be provided using a separate, secure online survey platform (www.unipark.de). All forms and data sets related to study data will be kept in locked cabinets. Study-related information will be stored securely in local, password-protected data files accessible only to the study team. Backups of the primary database (Unipark) will be performed weekly. Principal investigators will be given access to the processed data sets. Participant data will be identified by a coded identification number to maintain participant confidentiality. All reports will be prepared such that no individual subject can be identified. Figure 1 shows the study flow chart and inclusion criteria.

Primary outcome: somatic symptom distress

As a well-established and economic instrument, the PHQ- $15^{53}$ will be administered at each measurement 
point. The PHQ-15 is a validated brief self-report screening for somatoform disorders, ${ }^{62}$ including the 15 most typical somatic complaints in primary care. ${ }^{63}$ Participants rate the somatic symptom burden within the preceding 4 weeks on a 3 -point scale $(0=$ 'not bothered at all' to $2=$ 'bothered a lot'). The PHQ-15 has proven to be a reliable instrument $(\alpha=0.80){ }^{53}$

To account for the focus on dysfunctional psychological features of somatoform disorders as defined in the DSM-5, the recently developed Somatic Symptom Disorder Scale (SSD-12) was included.$^{64}$ The scale consists of 12 items covering the frequency of cognitive, affective and behavioural aspects in association with bothersome somatic symptoms. Answers are rated on a 5-point scale $(0=$ 'never' to $4=$ 'very often'). The instrument proved to be highly reliable in a clinical outpatient $(\alpha=0.95)^{64}$ and in a population-based sample $(\alpha=0.95){ }^{65}$

\section{Secondary outcomes}

To account for the high degree of co-occurrence between somatic symptom distress and symptoms of general and health anxiety as well as depression, ${ }^{46}$ these clinical comorbidities will be assessed with self-report instruments. The severity of depressive symptoms will be assessed with the 9-item Patient Health Questionnaire (PHQ-9) ${ }^{67}$ The nine items cover the DSM-IV criteria for major depression. Furthermore, the seven-item Generalised Anxiety Disorder Questionnaire (GAD-7) will be included to evaluate the severity of anxiety. Both instruments feature 4-point answer scales $(0=$ 'not at all' to $3=$ "nearly every day') and have proven to be valid screening and instruments in population-based samples. ${ }^{68} 69$ As a distinct feature of somatic symptom disorders, health anxiety will be assessed with a modified, 14-item short version of the Health Anxiety Inventory. ${ }^{70}$ Items (eg, "I am sometimes afraid I have a serious illness") are rated on a 5-point scale $(1=$ 'does not apply to me at all' to $4=$ 'does totally apply to me') and can be added to a total score, ranging from 0 to 56. The adapted version (modified short version of the Health Anxiety Inventory, mSHAI) proved to be highly reliable in a student population $(\alpha=0.93)$.

The level of disability will be assessed with the German version $^{71}$ of the Pain Disability Index (PDI) ${ }^{72}$ Participants rate the perceived disability on an 11-point scale $(0=$ 'no interference' to $10=$ 'total interference') in seven items representing various domains of functioning (eg, social activities, occupation, self-care). The scale shows an internal consistency of $\alpha=0.86 .^{73}$

\section{Attitudes and expectations}

The 10-item short form of the Attitudes Toward Seeking Professional Psychological Help questionnaire (ATSPPH $)^{74}$ will be included. Answers on this scale are rated on a 4-point scale ( $0=$ 'disagree' to $3=$ "agree'). The items (eg, "I would want to get psychological help if I were worried or upset for a long period of time") can be added to a total score, ranging from 0 to 30 . The ATSPPH shows a high reliability of $\alpha=0.84$.
To study the association of treatment expectations and outcomes, ${ }^{75}$ the Credibility/Expectancy Questionnaire $(\mathrm{CEQ})^{76}$ will be included. The questionnaire features six items (eg, "How successfully do you think this treatment will be in reducing your symptoms?"). The answer scale for two items (from $0 \%$ to $100 \%$ ) was adapted to correspond to the 9-point Likert scale of the remaining items. Higher scores indicate positive expectations and credibility. The CEQ has proven to be an easy-to-administer and reliable instrument $(\alpha=0.85)^{77}$ in clinical studies. Based on the previous literature on unfavourable attitudes of individuals with chronic somatic symptoms towards psychotherapy, ${ }^{78}$ we expect a positive influence of prior expectations on treatment outcomes as well as a positive change in attitudes towards psychotherapy in the prepost comparison.

\section{Subjective side effects}

Adverse events and side effects of the IMIs remain a less studied research field. ${ }^{79}$ Thus, adverse events during the intervention will be assessed with a 15 -item version of the Side Effects of Psychotherapy Inventory (INEP) ${ }^{80}$ as used in previous trials. ${ }^{81}$ The items cover possible adverse effects on social, intrapersonal or work-related situations (eg, 'worse/better relationship with family'). Six items are rated on a 7-point bipolar scale, the others on a 4-point Likert scale $(0=$ "no agreement at all'; $3=$ 'total agreement'). The original scale shows a high internal consistency of $\alpha=0.85$. Furthermore, symptom deterioration will be assessed as a possible negative effect of the intervention under study. ${ }^{79}$

\section{Therapeutic alliance}

Previous evidence suggests comparable alliance ratings by participants of guided IMIs to patients in face-to-face psychotherapy. ${ }^{82}{ }^{83}$ However, the association of perceived alliance and outcome remains inconclusive and the role of therapist's alliance ratings is insufficiently studied. ${ }^{84}$ Thus, in the present study, the therapeutic alliance will be assessed in the early intervention phase (3 weeks after intervention start) from participants and the associated e-coach with an adapted version of the short form of the Working Alliance Inventory (WAI-SR) ${ }^{85}$ as used in previous trials. ${ }^{86}$ The WAI is based on Bordin's three dimensions of working alliance ${ }^{87}$ : agreement on therapeutic goals, consensus on tasks, affective bond between client and therapist, which are assessed in four items each and rated on a 5 -point scale ( $1=$ 'never' to $5=$ 'always'). The version for the participants in the contact on demand condition will be adapted based on a previous study ${ }^{88}$ to measure alliance with the self-help programme.

\section{Intervention satisfaction and adherence}

The postintervention satisfaction will be assessed with a translated version of the Client Satisfaction Questionnaire (CSQ), adapted to the context of IMIs. ${ }^{89}$ The self-report questionnaire includes eight items that are rated on a 4 -point scale $(1=$ 'does not apply to me' to $4=$ 'does 
totally apply to me'). The adapted version proved to be highly reliable (McDonald's $\omega=0.95){ }^{89}$ Furthermore, acceptance of the intervention modules will be analysed using free feedback forms. Participant adherence to the intervention is assessed by the proportion of sessions completed during the intervention period and the total length (minutes) of the intervention exposure during the intervention period. Reasons for attrition will be assessed via email.

\section{Covariates}

Since previous research has demonstrated an effect-modifying influence of several demographic, clinical or internet-oriented variables,${ }^{90}$ the following covariates will be assessed: demographic variables (eg, age, gender, field of study), medical variables (eg, prior treatments, somatic/ mental diagnoses, current healthcare utilisation). Furthermore, we will assess the influence of somatosensory amplification, the tendency to experience somatic sensations as intense, noxious or disturbing ${ }^{91}$ with the 10-item German version of the Somatosensory Amplification Scale $(\mathrm{SSAS})^{92}$ as well as emotional reactivity, since somatisation has been associated with deficits in emotion regulation ${ }^{93}$ with a recently translated version of the Perth Emotion Reactivity Scale (PERS), ${ }^{94}$ including 30 items.

\section{Sample size estimation}

The estimation of the necessary sample size was based on power calculations and evidence from previous studies. The effect sizes of interventions for persistent somatic symptoms have a wide range and depend on the condition of intervention, that is, somatic symptom reduction in functional somatoform syndromes in guided iCBT with effect sizes from $\mathrm{d}=0.10^{95}$ to $\mathrm{d}=1.21,{ }^{96}$ aggregated effect sizes of self-help interventions in general (Hedge's $\mathrm{g}=0.58)^{15}$ or of face-to-face CBT $(\mathrm{d}=0.38) .{ }^{12}$ Based on these findings, a moderate effect size of $d=0.50$ is expected. A power analysis for the group comparison iSOMA versus WLCG using analysis of covariance (ANCOVA) with baseline scores as covariates, an $\alpha$ error of 0.05 and a power of $\beta=0.80$ showed that a sample size of $n=128$ is required. Considering attrition rates of previous trials, for example, in functional somatic syndromes, ${ }^{97}$ chronic pain ${ }^{98}$ or various chronic health conditions ${ }^{99}$ as well as recent trials in the relevant target group of university students, ${ }^{29}$ a drop-out rate of $20 \%$ is expected for the current trial, resulting in a necessary sample size of $n=154(n=77$ per group). The estimated sample size is suitable regarding prevalence rates of the somatoform syndrome ${ }^{45}$ and somatoform disorders in German university students. ${ }^{46}$

\section{Ethics and dissemination}

The methods described in this study protocol have been approved by the ethics committee of the Institute of Psychology of the University of Mainz (Ref. Nr. 2017-JGU-psychEK-012). Protocol modifications are subject to the ethics committee's approval and will be indicated in the trial registration. Results from this study will be published in peer-reviewed journals and presented at international conferences. Participant-friendly summaries of trial findings will be published on the project's website.

Before allocation to the trial, individuals receive detailed information about the nature, purpose and possible consequences of the trial. Participants are informed that the intervention under study does not replace medical and or psychotherapeutic treatment. Participants are required to give a written informed consent to participate in the study (model study information and consent form available on request).

Based on previous, similar trials, a very low risk of adverse events is expected. ${ }^{100} 101$ However, participants are able to contact the study team during working hours. Beyond that, participants are referred to a nation-wide and $24 \times 7$ telephone crisis hotline and receive detailed information about treatment options. If participants of both study arms indicate suicidal ideation in the Beck Depression Inventory-II (BDI-II) ${ }^{102}$ or within the intervention, a standardised suicide protocol adapted from previous trials in depressive populations ${ }^{55} 103$ will be applied. Participants are required to indicate their telephone number for emergency contact. (1) Participants who report a low level of suicidal ideation (BDI-II item score $=1$ ) will receive information with psychoeducation, information on health services via email and will be advised to seek professional help in case of symptom deterioration. (2) Participants with a moderate BDI-II item score (=2) additionally will receive a non-suicide contract and access to a separate web-based lecture on suicidality, where they will be guided in filling out an individual crisis plan and develop coping mechanisms. ${ }^{104}$ (3) If high levels of suicidal ideation are reported (BDI-II item score $=3$ ), participants will be contacted by phone by one of the supervisors $(\mathrm{SH}, \mathrm{MW})$ for risk assessment and to initiate further actions (eg, information, non-suicide contract, crisis plan, treatment options).

\section{Statistical analyses}

Intention-to-treat (ITT) samples will be analysed. Additional per-protocol analyses will be conducted to examine the influence of attrition on outcomes. No interim analyses will be conducted on the primary end point. Primary and secondary outcomes will be analysed as continuous variables (eg, sum scores). The primary and secondary outcomes at follow-up $\left(T_{1}, T 2\right)$ will be analysed with ANCOVA, controlling for the baseline measurement $\left(\mathrm{T}_{0}\right)$ and empirically identified covariates (significant zeroorder correlations with the primary outcome) according to recommendations by Vickers and Altman. ${ }^{105}$ Multiple regression models will be used to test potential mediating or moderating effects (eg, somatosensory amplification, emotional reactivity and therapeutic alliance). Additionally, to investigate the number of participants attaining a reliable improvement in the primary outcome measurement, scores will be characterised as responders, non-clinically changed or non-responders according 
to the reliable change index. ${ }^{106}$ Strategies for replacing missing data will be evaluated after the amount and pattern of missing data has been investigated. Subgroup analyses will be conducted after relevant characteristics of the final study sample have been investigated. Characteristics of dropouts at follow-up will be compared with responders. All analyses will be conducted on a two-sided level of significance $(\alpha=0.05)$.

\section{DISCUSSION}

To the best our knowledge, the proposed study is the first to investigate the efficacy of a CBT-based online intervention in an academic context for somatic symptoms and related distress. University students represent a highly relevant target group for preventive interventions since subclinical somatic syndromes are common ${ }^{45}$ and have been associated with serious clinical, occupational and societal consequences. ${ }^{45107}$ Furthermore, the evidence points to an undertreatment of this at-risk group. ${ }^{17} 107$ Since internet usage and acceptance of web-based health promotion is high in university students, ${ }^{25}{ }^{107}$ a web-based approach such as the studicare-framework represents an easy-accessible, international hub for low-threshold treatment options for various somatic and mental health issues. A recent trial investigating an IMI for stress in university students, for example, found that $77 \%$ of participants were first-time help-seekers. ${ }^{30}$ Therefore, the intervention under study represents a promising approach to prevent and to treat somatic symptoms and related distress on a public health level.

However, in conducting this trial, several challenges are expected and will be addressed as mentioned below.

First, to allow for low-threshold access, inclusion criteria will comprise somatic symptoms irrespective of their primary cause (physical or psychological), following the DSM-5 classification of SSD. Although the expected clinical diversity is welcome to test the generalisability of iSOMA as a transdiagnostic intervention, heterogeneity needs to be considered in further analyses. Therefore, subgroup analyses will be performed to differentiate the sample characteristics and evaluate a potential effect-modulating influence of comorbid somatic disorders.

Second, moderate-to-high drop-out rates have been reported for IMIs, that is, $4 \%^{96}$ to $38 \%^{108}$ in guided iCBT of IBS. Dropout may even be more pronounced in preventive oriented trials. The literature points to various risk factors for dropout, ${ }^{109-111}$ which were addressed in the development of iSOMA and the research design such as a short intervention duration ( 8 weeks), availability of guidance (e-coach, SMS-coach), ${ }^{28} 112$ reminders in case of inactivity ${ }^{58}$ and interactivity and variability of the intervention content (eg, multimedia-based, responsive design).${ }^{113}$ However, it needs to be considered that a reduced treatment adherence is not necessarily an indicator of limited effectiveness, but can also express early recovery. Thus, we attempt to assess the reasons for and timing of dropout in the present study.

Third, individuals with chronic somatic symptoms tend to develop somatically dominated models for their symptoms and thus may hold negative attitudes towards mental health interventions, ${ }^{19}{ }^{21}$ which could have implications for the accessibility of the intervention under study. Therefore, the intervention rationale ${ }^{50}$ features a primarily somatic-oriented entrance and a gradual development of a multicausal psychobiological explanatory model instead of an immediate introduction (table 1).

Previous trials focused on functional somatic syndromes or chronic pain, ${ }^{32} 33$ which leaves polysymptomatic conditions under-represented in research yet. However, international epidemiological studies show that somatic symptoms typically do not occur singularly but across multiple body systems. ${ }^{114115}$ Therefore, the current trial features a transdiagnostic approach considering the interplay of physiological and psychological symptoms in various clinical conditions. The strong theoretical foundation of intervention allows for directly targeting central dysfunctional mechanisms of the somatic syndrome. Thus, a higher effectiveness in comparison to non-manualised IMIs can be expected. ${ }^{116}$ Further distinct features of the intervention include the adaption of behavioural experiments and interoceptive exposure, which have been shown to be effective intervention components in IMIs. ${ }^{3796}$ Further strengths of this study include a rigorous methodical quality, including an ITT analysis, which prevents overestimation of the intervention's effectiveness. Also, various diagnostic instruments will be included to investigate possible moderators and mediators with a focus on psychopathological mechanisms, therapeutic alliance and potential side effects. In the future, this allows for a differentiated indication and identification of risk groups. Thus, the study results will have extensive implications for researchers, health professionals and academic mental healthcare.

\section{Author affiliations}

${ }^{1}$ Department of Clinical Psychology, Psychotherapy and Experimental

Psychopathology, Institute of Psychology, University of Mainz, Mainz, Germany

${ }^{2}$ Department of Clinical Psychology and Psychotherapy, Institute of Psychology and

Education, Ulm University, Ulm, Germany

${ }^{3}$ Department of Psychological Medicine, University of Auckland, Auckland, New Zealand

${ }^{4}$ Department of Psychology, Clinical Psychology and Psychotherapy, FriedrichAlexander University Erlangen Nuremberg, Erlangen, Germany

Acknowledgements The authors would like to thank Ann-Marie Küchler, Natalie Bauereiß (University of UIm), Lasse Sander (University of Freiburg) and Sylvan Germer (University of Mainz) for their considerable help in the planning of this study.

Contributors All authors were involved in the concept and design of the study. SH wrote the draft of this manuscript, $\mathrm{KB}, \mathrm{MW}, \mathrm{HB}, \mathrm{EB}, \mathrm{MK}$ and $\mathrm{DDE}$ provided valuable revisions. All authors contributed to the further writing and approved the final version of the manuscript.

Funding The proposed trial received funding for the development of video content by the Johannes Gutenberg-University of Mainz. The associated studicare-project received funding from the German health insurance company BARMER. 
Disclaimer The funding sources had no role in the design of this study and writing of this manuscript and will not have any role during its execution, analyses, interpretation of the data or decision to submit results.

Competing interests None declared.

Patient consent for publication Not required.

Ethics approval This study protocol has been approved by the ethics committee of the Institute of Psychology of the University of Mainz (Ref. Nr. 2017-JGU-psychEK-012).

Provenance and peer review Not commissioned; externally peer reviewed.

Open access This is an open access article distributed in accordance with the Creative Commons Attribution Non Commercial (CC BY-NC 4.0) license, which permits others to distribute, remix, adapt, build upon this work non-commercially, and license their derivative works on different terms, provided the original work is properly cited, appropriate credit is given, any changes made indicated, and the use is non-commercial. See: http://creativecommons.org/licenses/by-nc/4.0/.

\section{REFERENCES}

1. Henningsen $P$, Jakobsen $T$, Schiltenwolf M, et al. Somatization revisited: diagnosis and perceived causes of common mental disorders. J Nerv Ment Dis 2005;193:85-92.

2. den Boeft M, Twisk JWR, Terluin B, et al. The association between medically unexplained physical symptoms and health care use over two years and the influence of depressive and anxiety disorders and personality traits: a longitudinal study. BMC Health Serv Res 2016;16:100.

3. Budtz-Lilly A, Vestergaard M, Fink P, et al. The prognosis of bodily distress syndrome: a cohort study in primary care. Gen Hosp Psychiatry 2015;37:560-6.

4. Jacobi F, Wittchen $\mathrm{H}-\mathrm{U}$, Hölting $\mathrm{C}$, et al. Prevalence, co-morbidity and correlates of mental disorders in the general population: results from the German Health Interview and Examination Survey (GHS). Psychol Med 1999;34:597-611.

5. Larisch A, Fisch V, Fritzsche K. Kosten-Nutzen-Aspekte psychosozialer Interventionen bei somatisierenden Patienten in der Hausarztpraxis [Cost-effectiveness of psychosocial interventions for somatising patients by the general practitioner]. Z Klin Psychol Psychother 2005;34:282-90.

6. Rask MT, Rosendal M, Fenger-Grøn M, et al. Sick leave and work disability in primary care patients with recent-onset multiple medically unexplained symptoms and persistent somatoform disorders: a 10-year follow-up of the FIP study. Gen Hosp Psychiatry 2015;37:53-9.

7. Brown RJ. Psychological mechanisms of medically unexplained symptoms: an integrative conceptual model. Psychol Bull 2004;130:793-812.

8. van den Bergh $\mathrm{O}$, Witthöft M, Petersen S, et al. Symptoms and the body: Taking the inferential leap. Neurosci Biobehav Rev 2017;74:185-203.

9. van den Bergh $\mathrm{O}$, Winters W, Devriese $\mathrm{S}$, et al. Learning subjective health complaints. Scand J Psychol 2002;43:147-52.

10. Sattel H, Schaefert R, Häuser W, et al. [Treatment of non-specific, functional and somatoform bodily complaints]. Dtsch Med Wochenschr 2014:139:155-74.

11. van Dessel N, Den Boeft M, van der Wouden JC, et al. Nonpharmacological interventions for somatoform disorders and medically unexplained physical symptoms (MUPS) in adults, a Cochrane systematic review. J Psychosom Res 2015;78:628.

12. Kleinstäuber M, Witthöft M, Hiller W. Efficacy of short-term psychotherapy for multiple medically unexplained physical symptoms: A meta-analysis. Clin Psychol Rev 2011;31:146-60.

13. Henningsen P, Zipfel S, Herzog W. Management of functional somatic syndromes. The Lancet 2007;369:946-55.

14. Kleinstäuber $M$, Witthöft $M$, Steffanowski $A$, et al. Pharmacological interventions for somatoform disorders in adults. Cochrane Database Syst Rev 2014;32.

15. van Gils A, Schoevers RA, Bonvanie IJ, et al. Self-help for medically unexplained symptoms: a systematic review and meta-analysis. Psychosom Med 2016;78:728-39.

16. Wittchen $\mathrm{H}-U$, Jacobi F. Met and unmet needs for intervention. clinical-epidemiological estimations for mental disorders in the german health interview and examination survey supplement]. Bundesgesundheitsbl - Gesundheitsforsch - Gesundheitsschutz 2001;44:993-1000.
17. Auerbach RP, Alonso J, Axinn WG, et al. Mental disorders among college students in the World Health Organization World Mental Health Surveys. Psychol Med 2016;46:2955-70.

18. Mack S, Jacobi F, Gerschler A, et al. Self-reported utilization of mental health services in the adult German population--evidence for unmet needs? Results of the DEGS1-Mental Health Module (DEGS1-MH). Int J Methods Psychiatr Res 2014;23:289-303.

19. McGorm K, Burton C, Weller D, et al. Patients repeatedly referred to secondary care with symptoms unexplained by organic disease: prevalence, characteristics and referral pattern. Fam Pract 2010;27:479-86.

20. Eisenberg D, Downs MF, Golberstein E, et al. Stigma and help seeking for mental health among college students. Med Care Res Rev 2009;66:522-41.

21. Freidl M, Spitzl SP, Prause W, et al. The stigma of mental illness: anticipation and attitudes among patients with epileptic, dissociative or somatoform pain disorder. Int Rev Psychiatry 2007;19:123-9.

22. Hahn SR, Kroenke K, Spitzer RL, et al. The difficult patient. J Gen Intern Med 1996;11:1-8.

23. Paganini S, Teigelkötter W, Buntrock C, et al. Economic evaluations of internet- and mobile-based interventions for the treatment and prevention of depression: A systematic review. J Affect Disord $2018 ; 225$

24. Ebert DD, Van Daele T, Nordgreen T, et al. Internet- and mobilebased psychological interventions: applications, efficacy, and potential for improving mental health. Eur Psychol 2018;23:167-87.

25. Matusiewicz D, Krol B, Stender T, et al. Health Report of professional students: Analysis of health promotion in study and occupation, the willingness to pay and the use of digital media]. Gesundheitsökonomie \& Qualitätsmanagement 2017.

26. Chan JK, Farrer LM, Gulliver A, et al. University students' views on the perceived benefits and drawbacks of seeking help for mental health problems on the internet: a qualitative study. JMIR Hum Factors 2016;3:e3.

27. Ebert DD, Cuijpers P, Muñoz RF, et al. Prevention of mental health disorders using internet- and mobile-based interventions: a narrative review and recommendations for future research. Front Psychiatry 2017;8:116.

28. Baumeister $\mathrm{H}$, Reichler L, Munzinger M, et al. The impact of guidance on Internet-based mental health interventions - A systematic review. Internet Interv 2014;1:205-15.

29. Davies EB, Morriss R, Glazebrook C. Computer-delivered and web-based interventions to improve depression, anxiety, and psychological well-being of university students: a systematic review and meta-analysis. J Med Internet Res 2014;16:e130.

30. Harrer M, Adam SH, Fleischmann RJ, et al. Effectiveness of an internet- and app-based intervention for college students with elevated stress: randomized controlled trial. J Med Internet Res 2018;20:e136.

31. van Beugen S, Ferwerda M, Hoeve D, et al. Internet-based cognitive behavioral therapy for patients with chronic somatic conditions: a meta-analytic review. J Med Internet Res 2014;16:e88.

32. McCombie A, Gearry R, Andrews J, et al. Computerised cognitive behavioural therapy for psychological distress in patients with physical illnesses: a systematic review. J Clin Psychol Med Settings 2015;22:20-44.

33. Beatty L, Lambert S. A systematic review of internet-based selfhelp therapeutic interventions to improve distress and diseasecontrol among adults with chronic health conditions. Clin Psychol Rev 2013;33:609-22.

34. Toivonen KI, Zernicke K, Carlson LE. Web-based mindfulness interventions for people with physical health conditions: systematic review. J Med Internet Res 2017;19:e303.

35. Liegl G, Plessen CY, Leitner A, et al. Guided self-help interventions for irritable bowel syndrome: a systematic review and metaanalysis. Eur J Gastroenterol Hepatol 2015;27:1209-21.

36. Piga M, Cangemi I, Mathieu A, et al. Telemedicine for patients with rheumatic diseases: Systematic review and proposal for research agenda. Semin Arthritis Rheum 2017:47:121-8.

37. Hedman E, Andersson G, Andersson E, et al. Internet-based cognitive-behavioural therapy for severe health anxiety: randomised controlled trial. Br J Psychiatry 2011;198:230-6.

38. Macea DD, Gajos K, Daglia Calil YA, et al. The efficacy of Webbased cognitive behavioral interventions for chronic pain: a systematic review and meta-analysis. J Pain 2010;11:917-29.

39. Hedman E, Axelsson E, Andersson E, et al. Exposure-based cognitive-behavioural therapy via the internet and as bibliotherapy for somatic symptom disorder and illness anxiety disorder: randomised controlled trial. British Journal of Psychiatry 2016;209:407-13. 
40. Newby JM, Smith J, Uppal S, et al. Internet-based cognitive behavioral therapy versus psychoeducation control for illness anxiety disorder and somatic symptom disorder: A randomized controlled trial. J Consult Clin Psychol 2018;86:89-98.

41. Leiknes KA, Finset A, Moum T, et al. Current somatoform disorders in Norway: prevalence, risk factors and comorbidity with anxiety, depression and musculoskeletal disorders. Soc Psychiatry Psychiatr Epidemiol 2007;42:698-710.

42. Berger $\mathrm{H}$, Franke $\mathrm{GH}$, Hofmann F-H, et al. Mental health of students and its development between 1994 and 2012. Ment Health Prev 2015;3(1-2):48-56.

43. Hunt J, Eisenberg D. Mental health problems and help-seeking behavior among college students. J Adolesc Health 2010;46:3-10.

44. Heilmann VK, Brähler E, Hinz A, et al. [Psychological distress, need for advice and utilization of professional help among students]. Psychother Psychosom Med Psychol 2015;65(34):99-103.

45. Bailer J, Schwarz D, Witthöft M, et al. [Prevalence of mental disorders among college students at a German university]. Psychother Psychosom Med Psychol 2008;58:423-9.

46. Grobe T, Steinmann S. Gesundheitsreport 2015. Gesundheit von Studierenden. 2015. https://www.tk.de/centaurus/servlet/ contentblob/718612/Datei/143833/Gesundheitsreport-2015.pdf (accessed 25 Sep 17).

47. Robotham D, Julian C. Stress and the higher education student: a critical review of the literature. Journal of Further and Higher Education 2006;30:107-17.

48. Cook LJ. Striving to help college students with mental health issues. J Psychosoc Nurs Ment Health Serv 2007;45:40-4

49. Bruffaerts R, Mortier P, Kiekens G, et al. Mental health problems in college freshmen: Prevalence and academic functioning. J Affect Disord 2018;225:97-103.

50. Kleinstäuber M, Thomas P, Witthöft M, et al. Cognitive Behavioral Therapy for Medically Unexplained Physical Symptoms and Somatoform Disorders]. Berlin: Springer 2018.

51. Schulz KF, Altman DG, Moher D. CONSORT 2010 Statement: updated guidelines for reporting parallel group randomised trials. BMC Med 2010;8:18.

52. Proudfoot J, Klein B, Barak A, et al. Establishing guidelines for executing and reporting internet intervention research. Cogn Behav Ther 2011;40:82-97.

53. Kroenke K, Spitzer RL, Williams JBW. The PHQ-15: validity of a new measure for evaluating the severity of somatic symptoms. Psychosom Med 2002;64:258-66.

54. Laferton JAC, Stenzel NM, Rief W, et al. Screening for dsm-5 somatic symptom disorder: diagnostic accuracy of self-report measures within a population sample. Psychosom Med 2017.

55. Sander L, Paganini S, Lin J, et al. Effectiveness and costeffectiveness of a guided Internet- and mobile-based intervention for the indicated prevention of major depression in patients with chronic back pain - study protocol of the PROD-BP multicenter pragmatic RCT. BMC Psychiatry 2017;17:36.

56. Ebert DD, Nobis S, Lehr D, et al. The 6-month effectiveness of Internet-based guided self-help for depression in adults with Type 1 and 2 diabetes mellitus. Diabetic Medicine 2017;34:99-107.

57. Hilvert-Bruce Z, Rossouw PJ, Wong N, et al. Adherence as a determinant of effectiveness of internet cognitive behavioural therapy for anxiety and depressive disorders. Behav Res Ther 2012;50(7-8):463-8.

58. Fry JP, Neff RA. Periodic prompts and reminders in health promotion and health behavior interventions: systematic review. $J$ Med Internet Res 2009;11:e16.

59. Eckert M, Ebert DD, Lehr D, et al. Does sms-support make a difference? effectiveness of a two-week online-training to overcome procrastination. a randomized controlled Trial. Front Psychol 2018;9:1103.

60. Berger T, Caspar F, Richardson R, et al. Internet-based treatment of social phobia: A randomized controlled trial comparing unguided with two types of guided self-help. Behav Res Ther 2011;49:158-69 http://www.sciencedirect.com/science/article/pii/ S0005796710002561.

61. Janse A, Worm-Smeitink M, Bleijenberg G, et al. Efficacy of webbased cognitive-behavioural therapy for chronic fatigue syndrome: randomised controlled trial. The British Journal of Psychiatry 2018;212:112-8.

62. Kroenke K, Spitzer RL, Williams JBW, et al. The Patient Health Questionnaire Somatic, Anxiety, and Depressive Symptom Scales: a systematic review. Gen Hosp Psychiatry 2010;32:345-59.

63. Spitzer RL, et al. Utility of a new procedure for diagnosing mental disorders in primary care. JAMA 1994;272:1749-56.
64. Toussaint A, Murray AM, Voigt K, et al. Development and validation of the somatic symptom disorder-b criteria scale (SSD-12). Psychosom Med 2016;78:5-12.

65. Toussaint A, Löwe B, Brähler E, et al. The Somatic Symptom Disorder - B Criteria Scale (SSD-12): Factorial structure, validity and population-based norms. J Psychosom Res 2017;97:9-17.

66. Ansseau M, Dierick M, Buntinkx F, et al. High prevalence of mental disorders in primary care. J Affect Disord 2004;78:49-55.

67. Kroenke K, Spitzer RL. The PHQ-9: A new depression diagnostic and severity measure. Psychiatr Ann 2002;32:509-15.

68. Martin A, Rief W, Klaiberg A, et al. Validity of the Brief Patient Health Questionnaire Mood Scale (PHQ-9) in the general population. Gen Hosp Psychiatry 2006;28:71-7.

69. Löwe B, Decker O, Müller S, et al. Validation and standardization of the Generalized Anxiety Disorder Screener (GAD-7) in the general population. Med Care 2008;46:266-74.

70. Bailer J, Witthöft M. Deutsches modifiziertes health anxiety inventory (MK-HAI)]. Zusammenstellung sozialwissenschaftlicher Items und Skalen 2014

71. Dillmann $U$, Nilges $P$, Saile $H$, et al. [Assessing disability in chronic pain patients.]. Schmerz 1994;8:100-10.

72. Pollard CA. Preliminary validity study of the pain disability index. Percept Mot Skills 1984;59:974.

73. Tait RC, Chibnall JT, Krause S. The Pain Disability Index: psychometric properties. Pain 1990;40:171-82.

74. Picco L, Abdin E, Chong SA, et al. Attitudes toward seeking professional psychological help: factor structure and sociodemographic predictors. Front Psychol 2016;7.

75. Titov N, Andrews G, Choi I, et al. Shyness 3: randomized controlled trial of guided versus unguided Internet-based CBT for social phobia. Aust N Z J Psychiatry 2008;42:1030-40.

76. Borkovec TD, Nau SD. Credibility of analogue therapy rationales. $J$ Behav Ther Exp Psychiatry 1972;3:257-60.

77. Devilly GJ, Borkovec TD. Psychometric properties of the credibility/expectancy questionnaire. J Behav Ther Exp Psychiatry 2000;31:73-86.

78. Timmer B, Bleichhardt G, Rief W. Importance of Psychotherapy motivation in patients with somatization syndrome. Psychotherapy Research 2006;16:348-56.

79. Rozental A, Andersson G, Boettcher J, et al. Consensus statement on defining and measuring negative effects of Internet interventions. Internet Interv 2014;1:12-19.

80. Ladwig I, Rief W, Nestoriuc Y. What Are the Risks and Side Effects of Psychotherapy? - Development of an Inventory for the Assessment of Negative Effects of Psychotherapy (INEP). Verhaltenstherapie 2014;24:252-63.

81. Boß L, Lehr D, Berking M, et al. Evaluating the (cost-)effectiveness of guided and unguided Internet-based self-help for problematic alcohol use in employees--a three arm randomized controlled trial. BMC Public Health 2015;15:1043.

82. Ertelt TW, Crosby RD, Marino JM, et al. Therapeutic factors affecting the cognitive behavioral treatment of bulimia nervosa via telemedicine versus face-to-face delivery. Int J Eat Disord 2011;44:687-91.

83. Kiropoulos LA, Klein B, Austin DW, et al. Is internet-based CBT for panic disorder and agoraphobia as effective as face-to-face CBT? J Anxiety Disord 2008;22:1273-84.

84. Berger T. The therapeutic alliance in internet interventions: A narrative review and suggestions for future research. Psychother Res 2017;27:511-24.

85. Wilmers F, Munder T, Leonhart R, et al. Die deutschsprachige Version des Working Alliance Inventory - short revised (WAI-SR) Ein schulenübergreifendes, ökonomisches und empirisch validiertes Instrument zur Erfassung der therapeutischen Allianz. Klinische Diagnostik und Evaluation 2008;1:343-58.

86. Jasper $\mathrm{K}$, Weise $\mathrm{C}$, Conrad I, et al. The working alliance in a randomized controlled trial comparing Internet-based self-help and face-to-face cognitive behavior therapy for chronic tinnitus. Internet Interv 2014;1:49-57.

87. Bordin ES. The generalizability of the psychoanalytic concept of the working alliance. Psychotherapy: Theory, Research \& Practice 1979;16:252-60.

88. Berger T, Boettcher J, Caspar F. Internet-based guided self-help for several anxiety disorders: a randomized controlled trial comparing a tailored with a standardized disorder-specific approach. Psychotherapy 2014;51:207-19.

89. Boß L, Lehr D, Reis D, et al. Reliability and validity of assessing user satisfaction with web-based health interventions. J Med Internet Res 2016;18:e234.

90. Ebert DD, Gollwitzer M, Riper H, et al. For whom does it work? moderators of outcome on the effect of a transdiagnostic internet- 
based maintenance treatment after inpatient psychotherapy: randomized controlled trial. J Med Internet Res 2013;15:e191.

91. Barsky AJ, Goodson JD, Lane RS, et al. The amplification of somatic symptoms. Psychosom Med 1988;50:510-9.

92. Rief W, Hiller W, Margraf J. Cognitive aspects of hypochondriasis and the somatization syndrome. J Abnorm Psychol 1998;107:587-95.

93. Schwarz J, Rief W, Radkovsky A, et al. Negative affect as mediator between emotion regulation and medically unexplained symptoms. J Psychosom Res 2017;101:114-21.

94. Becerra R, Campitelli G. Emotional reactivity: critical analysis and proposal of a new scale. Int J Appl Psychol 2013;3:161-8.

95. Lorig KR, Ritter PL, Laurent DD, et al. The internet-based arthritis self-management program: a one-year randomized trial for patients with arthritis or fibromyalgia. Arthritis Rheum 2008;59:1009-17.

96. Ljótsson B, Falk L, Vesterlund AW, et al. Internet-delivered exposure and mindfulness based therapy for irritable bowel syndrome--a randomized controlled trial. Behav Res Ther 2010;48:531-9.

97. Bernardy K, Klose P, Welsch P, et al. Efficacy, acceptability and safety of Internet-delivered psychological therapies for fibromyalgia syndrome: A systematic review and meta-analysis of randomized controlled trials. Eur J Pain 2018.

98. Buhrman M, Gordh T, Andersson G. Internet interventions for chronic pain including headache: A systematic review. Internet Interv 2016;4:17-34.

99. Cuijpers P, van Straten A, Andersson G. Internet-administered cognitive behavior therapy for health problems: a systematic review. J Behav Med 2008;31:169-77.

100. Boettcher J, Rozental A, Andersson G, et al. Side effects in Internetbased interventions for Social Anxiety Disorder. Internet Interv 2014;1:3-11.

101. Rozental A, Boettcher J, Andersson G, et al. Negative effects of internet interventions: a qualitative content analysis of patients' experiences with treatments delivered online. Cogn Behav Ther 2015;44:223-36.

102. Beck AT, Steer RA, Brown G. Beck Depression Inventory-II. PsycTESTS Dataset The Psychological Corporation, San Antonio 1996.

103. Buntrock C, Ebert D, Lehr D, et al. Effectiveness of a web-based cognitive behavioural intervention for subthreshold depression: pragmatic randomised controlled trial. Psychother Psychosom 2015;84:348-58.
104. Teismann T, Dorrmann W. Suicidality. Berlin: Hogrefe, 2014.

105. Vickers AJ, Altman DG. Statistics notes: Analysing controlled trials with baseline and follow up measurements. BMJ 2001;323:1123-4.

106. Jacobson NS, Truax P. Clinical significance: a statistical approach to defining meaningful change in psychotherapy research. J Consult Clin Psychol 1991;59:12-19.

107. Ryan ML, Shochet IM, Stallman HM. Universal online interventions might engage psychologically distressed university students who are unlikely to seek formal help. Advances in Mental Health 2010;9:73-83.

108. Hunt MG, Moshier S, Milonova M. Brief cognitive-behavioral internet therapy for irritable bowel syndrome. Behav Res Ther 2009;47:797-802.

109. Morrison LG, Yardley L, Powell J, et al. What design features are used in effective e-health interventions? A review using techniques from Critical Interpretive Synthesis. Telemed J E Health 2012;18:137-44.

110. Donkin L, Glozier N. Motivators and motivations to persist with online psychological interventions: a qualitative study of treatment completers. J Med Internet Res 2012;14:e91.

111. Kelders SM, Kok RN, Ossebaard HC, et al. Persuasive system design does matter: a systematic review of adherence to webbased interventions. J Med Internet Res 2012;14:e152.

112. Lindhiem O, Bennett CB, Rosen D, et al. Mobile technology boosts the effectiveness of psychotherapy and behavioral interventions: a meta-analysis. Behav Modif 2015;39:785-804.

113. Höffler TN, Leutner D. Instructional animation versus static pictures: A meta-analysis. Learn Instr 2007;17:722-38.

114. Hiller W, Rief W, Brähler E. Somatization in the population: from mild bodily misperceptions to disabling symptoms. Soc Psychiatry Psychiatr Epidemiol 2006;41:704-12.

115. McAteer A, Elliott AM, Hannaford PC. Ascertaining the size of the symptom iceberg in a UK-wide community-based survey. Br J Gen Pract 2011;61:e1-e11.

116. Webb TL, Joseph J, Yardley L, et al. Using the internet to promote health behavior change: a systematic review and meta-analysis of the impact of theoretical basis, use of behavior change techniques, and mode of delivery on efficacy. J Med Internet Res 2010;12:e4.

117. Lazarus RS, Launier R. Stress-related transactions between person and environment. Perspectives in interactional psychology. New York: Plenum Press, 1978:287-327. 